\title{
THE ISOPERIMETRIC INEQUALITY ON ASYMPTOTICALLY FLAT MANIFOLDS WITH NONNEGATIVE SCALAR CURVATURE
}

\author{
YUGUANG SHI
}

\begin{abstract}
In this note, we consider the isoperimetric inequality on an asymptotically flat manifold with nonnegative scalar curvature, and improve it by using Hawking mass. We also obtain a rigidity result when equality holds for the classical isoperimetric inequality on an asymptotically flat manifold with nonnegative scalar curvature.
\end{abstract}

\section{INTRODUCTION}

The isoperimetric inequality and isoperimetric surfaces have a very long history and many important applications in mathematics, see e.g. [1], 4]. Huisken has observed that ADM mass of an asymptotically flat manifold (see Definition 1.1 below) appears in the expansion of isoperimetric ratio when the volume is large enough, see [9] and [6] (for the case of coordinates sphere, see [7]). Inspired by these facts, it is natural to ask if there is any relationship between the isoperimetric inequality and quasi-local mass for any fixed enclosed volume. In this short note, we are able to use the Hawking mass to improve the isoperimetric inequality in some cases. In order to present our result, we need some notions.

Definition 1.1. A complete and connected three-manifold $\left(M^{3}, g\right)$ is said to be asymptotically flat $(A F)$ (with one end) if there are a positive constant $C>0$ and a compact subset $K$ such that $M \backslash K$ is diffeomorphic to $\mathbb{R}^{3} \backslash B_{R}(0)$ for some $R>0$ and in the standard coordinates in $\mathbb{R}^{3}$, the metric $g$ satisfies:

$$
g_{i j}=\delta_{i j}+\sigma_{i j}
$$

with

$$
\left|\sigma_{i j}\right|+r\left|\partial \sigma_{i j}\right|+r^{2}\left|\partial \partial \sigma_{i j}\right| \leq C r^{-1}
$$

Date: 2015 .

2000 Mathematics Subject Classification. Primary 83C57 ; Secondary 53C44.

Key words and phrases. isoperimetric inequality; inverse mean curvature flow; Hawking mass; asymptotically flat manifold .

$\dagger$ Research partially supported by NSF grant of China 10725101 and 10990013. 
where $r$ and $\partial$ denote the Euclidean distance and standard derivative operator on $\mathbb{R}^{3}$ respectively. The region $M \backslash K$ is called the end of $M$.

An original idea of [2] is to use the weak solution of inverse mean curvature (4) to estimate the volumes of isoperimetric regions in an asymptotically hyperbolic manifold. Inspired by this, we use the same idea to investigate the same problem in the case of AF manifolds. More specifically, for any $x \in M$, it is proved here that there is a weak solution $\left(G_{t}\right)_{t>-\infty}$ of (44) with initial condition $\{x\}$ in [8]. One important property for this weak solution is that for each $t \in \mathbb{R},\left(G_{t}\right)$ has the least boundary area among all domains containing it, i.e. $\left(G_{t}\right)$ is a minimizing hull in $\left(M^{3}, g\right)$. Another interesting property is that the Hawking mass of $K_{t}=\partial G_{t}$ which is defined as

$$
m_{H}(t)=\frac{\left(\operatorname{Area}\left(K_{t}\right)\right)^{\frac{1}{2}}}{(16 \pi)^{\frac{3}{2}}}\left(16 \pi-\int_{K_{t}} H^{2}\right),
$$

is nondecreasing in $t$; here, $H$ is the mean curvature of $K_{t}(x)=\partial G_{t}$ with respect to outward unit normal vector. By using this quantity, we are able to estimate the area of $K_{t}$ in terms of the volume of $G_{t}$, see (15) below; hence, we obtain Theorem 1.2. To do that, we need to parametrize $t$ by $v$, which is the volume of $G_{t}$, and it turns out that this function $t(v)$ is Lipschitz; for details, see Lemma 3.4 below. Let $m(v)=m_{H}(t(v)), B(v)=\operatorname{Area}\left(K_{t(v)}\right)$, and

$$
\begin{gathered}
A(v)=\inf \left\{\mathcal{H}^{2}\left(\partial^{*} \Omega\right): \Omega \subset M\right. \text { is a Borel set with finite perimeter, and } \\
\left.\mathcal{L}^{3}(\Omega)=v\right\} .
\end{gathered}
$$

here, $\mathcal{H}^{2}$ is 2-dimensional Hausdorff measure for the reduced boundary of $\Omega$, and $\mathcal{L}^{3}(\Omega)$ is the Lebesgue measure of $\Omega$ with respect to metric $g$.

Then our main result can be stated as follows

Theorem 1.2. Suppose $\left(M^{3}, g\right)$ is an asymptotically flat $(A F)$ manifold with nonnegative scalar curvature. Fix a point $o \in M$, for every $v>0$, there is a $\rho>0$ so that for all $x \in M \backslash B_{\rho}(0)$ we have that

$$
A(v) \leq(36 \pi)^{\frac{1}{3}}\left(\int_{0}^{v}\left(1-(16 \pi)^{\frac{1}{2}} B^{-\frac{1}{2}}(t) m(t)\right)^{\frac{1}{2}} d t\right)^{\frac{2}{3}} .
$$

Where $m(v)$ is defined as above.

When scalar curvature of $M$ is non-negative, and $M$ satisfies some topological conditions, we have that $m(v) \geq 0$. We see that in this case $A(v) \leq$ $(36 \pi)^{\frac{1}{3}} v^{\frac{2}{3}}$. Comparing this with the Euclidean case in which $m(v)=0$, we observe the following heuristic phenomenon: to enclose the same volume, 
isoperimetric surfaces in a manifold with bigger mass have smaller area. We believe such a phenomenon can also be observed in the case of asymptotically hyperbolic manifolds, and we will discuss this problem in a future paper. With these facts in mind, it is natural to ask what happens if there is a $v_{0}>0$ with $A\left(v_{0}\right)=(36 \pi)^{\frac{1}{3}} v_{0}^{\frac{2}{3}}$ ? Our next theorem gives an answer to this question.

Theorem 1.3. Suppose $\left(M^{3}, g\right)$ is an asymptotically flat manifold with nonnegative scalar curvature. Then there is a $v_{0}>0$ with

$$
A\left(v_{0}\right)=(36 \pi)^{\frac{1}{3}} v_{0}^{\frac{2}{3}}
$$

if and only if $\left(M^{3}, g\right)$ is isometric to $\mathbb{R}^{3}$.

Inequality (15) below is crucial in the proof of Theorem 1.2 and Theorem 1.3, and its equivalent version was first proved in [2] (see Proposition 3 in [2]), and the arguments here are quite similar to those in [2].

Theorem 1.2 and Theorem 1.3 play important roles in the proof of existence of isoperimetric regions in a non-flat AF manifold. There are many results that focus on large isoperimetric regions in an AF manifold, where the asymptotic regime plays an important role, see [5] and references therein. However, there are very few results on the existence of isoperimetric regions with medium size in an AF manifold. One difficulty is that the minimizing sequence of isoperimetric regions may drift off to infinity, while Theorem 1.2 allows for control over these minimizing sequence in a certain sense and we may obtain the existence isoperimetric regions with any given volume. This was observed in the very recent paper [3].

The outline of the paper is as follows. In Section 2, we introduce some notions and basic facts of weak solutions of inverse mean curvature flow from [8]; in Section 3, we prove the main results.

Acknowledgements The author is grateful to Prof. Frank Morgan, Dr. Gang Li and Dr. Chao Bao for pointing out some typos and errors in the first version of the paper, and also would like to thank referees for many useful comments and suggestions which make the paper clearer. Especially, the author would like to thank one of referees for pointing out that the assumptions of Theorem 1.2 and Theorem 1.3 can be relaxed by considering IMCF in the exterior region of $M$.

\section{Preliminary}

In this section, we introduce some notions and present some facts from [8] that will be needed in the proof of Theorem 1.2 and Theorem 1.3. As in [8], a classical solution of the inverse mean curvature flow (IMCF) in $\left(M^{3}, g\right)$ is a smooth family of $F: N \times[0, T] \rightarrow M$ of embedded hypersurfaces 
$N_{t}=F(N, t)$ satisfying the following evolution equation

$$
\frac{\partial F}{\partial t}=H^{-1} \nu, \quad 0 \leq t \leq T
$$

where $H$ is the mean curvature of $N_{t}$ at $F(x, t)$ with respect to the outward unit normal vector $\nu$ for any $x \in N$. In generally, the evolution equation (4) has no classical solution. In order to overcome this difficult, a level set approach was established in [8], i.e. these evolving surfaces were given as the level-sets of a scalar function $u$ via $N_{t}=\partial\{x \in M: u(x)<t\}$, where $u$ satisfies the following degenerate elliptic equation in weak sense.

$$
\operatorname{div}_{M}\left(\frac{\nabla u}{|\nabla u|}\right)=|\nabla u|
$$

Here the left-hand side describes the mean curvature of level-sets and the right-hand side yields the inverse speed.

By the definition of AF manifolds, for any $x \in M \backslash K$, we may consider standard coordinates $x=\left(x^{1}, x^{2}, x^{3}\right)$ on $\mathbb{R}^{3}$. It was observed in [8] that $v(x)=C \log |x|$ is a weak subsolution of (5) on $M \backslash K$ (please see the precise definition of weak subsolution of (5) on P.365 in [8] ), where $|x|=\sqrt{\left(x^{1}\right)^{2}+\left(x^{2}\right)^{2}+\left(x^{3}\right)^{2}}$. With this weak subsolution one is able to prove the existence of the weak solution of (5) on $M$ with any nonempty precompact smooth open set $E_{0}$ as initial condition (See Theorem 3.1 in [8]). Let $u^{\epsilon}$ be the solution of the following elliptic regularization:

$$
\begin{cases}E^{\epsilon} u^{\epsilon}=\operatorname{div}\left(\frac{\nabla u^{\epsilon}}{\sqrt{\left|\nabla u^{\epsilon}\right|^{2}+\epsilon^{2}}}\right)-\sqrt{\left|\nabla u^{\epsilon}\right|^{2}+\epsilon^{2}}=0, & \text { in } \Omega_{L} \\ u^{\epsilon}=0, & \text { on } \partial E_{0} \\ u^{\epsilon}=L-2, & \text { on } \partial F_{L}\end{cases}
$$

Here and in the sequel, $F_{L}=\{v<L\}$, for any large $L>0$, and $\Omega_{L}=$ $F_{L} \backslash \bar{E}_{0}$, let $W^{\epsilon}(x, z)=u^{\epsilon}(x)-\epsilon z$ be a function on $\Omega_{L} \times \mathbb{R}$, then we have

$$
\operatorname{div}\left(\frac{\nabla W^{\epsilon}}{\left|\nabla W^{\epsilon}\right|}\right)=\left|\nabla W^{\epsilon}\right|
$$

or equivalently, the level set $N_{t}^{\epsilon}=\left\{(x, z) \in \Omega_{L} \times \mathbb{R}: W^{\epsilon}(x, z)=t\right\}$ is a slice of the inverse mean curvature flow in the domain $\Omega_{L} \times \mathbb{R}$ for any $t>0$, and actually it is the classical solution to (44). We know from Lemma 3.5 in [8] that (6) admits a classical solution. Also, we have the following compactness lemma and its proof can be found on P.398 in [8].

Lemma 2.1. Let $\left(M^{3}, g\right)$ be an $A F$ manifold, and $E_{0}$ be a precompact set of $M$ with smooth boundary. Then there are subsequences $\epsilon_{i} \rightarrow 0, L_{i} \rightarrow \infty$, 
$N_{t}^{i}=N_{t}^{\epsilon_{i}}$ such that

$$
N_{t}^{i} \rightarrow \tilde{N}_{t}=N_{t} \times \mathbb{R}, \quad \text { locally in } C^{1}, \quad \text { for almost every } t \geq 0
$$

where $N_{t}=\partial E_{t}$ and $\left(E_{t}\right)_{t>0}$ is the unique weak solution of (4) with $E_{0}$ as the initial condition.

\section{Proof of THE MAIN THEOREMS}

In this section, we first establish some lemmas, and then prove our main results. Many arguments are from [2]. Lemma 3.4 below plays an important role in the proof, and meanwhile we note that many quantities involved are not smooth along the weak solution of inverse mean curvature flow (44). To handle this difficulty, we first calculate the corresponding quantities along the solutions to elliptic regularizations with suitable boundary conditions. Passing to the limit using Lemma 2.1, we get what we want.

Let $B_{\mu}(x)$ be any geodesic ball with radius $\mu>0$ and center $x$ in $(M, g)$, and let $E_{0}=B_{\mu}(x)$. We consider the following boundary problem

$$
\begin{cases}E^{\epsilon} u^{\epsilon}=\operatorname{div}\left(\frac{\nabla u^{\epsilon}}{\sqrt{\left|\nabla u^{\epsilon}\right|^{2}+\epsilon^{2}}}\right)-\sqrt{\left|\nabla u^{\epsilon}\right|^{2}+\epsilon^{2}}=0, & \text { in } \Omega_{L} \\ u^{\epsilon}=0, & \text { on } \partial E_{0} \\ u^{\epsilon}=L-2, & \text { on } \partial F_{L} .\end{cases}
$$

Using Lemma 2.1, we know there are subsequences $\epsilon_{i} \rightarrow 0, L_{i} \rightarrow \infty$, $N_{t}^{i}=N_{t}^{\epsilon_{i}}$ such that

$$
N_{t}^{i} \rightarrow \tilde{N}_{t}=N_{t} \times \mathbb{R}, \quad \text { locally in } C^{1}, \text { for almost every } t \geq 0
$$

where $N_{t}=\partial E_{t}$ and $\left(E_{t}\right)_{t>0}$ is the unique weak solution of (4) with the initial condition $E_{0}=B_{\mu}(x)$. For simplicity, as in the proof of Lemma 8.1 in [8], for each $\mu>0$, we may take a suitable transformation on $t$, so that the weak solution $\left(E_{t}\right)$ for the initial value problem (4) is defined on $[-T(\mu), \infty)$. Here $T(\mu) \rightarrow \infty$ as $\mu$ approaches to zero, and $\left(E_{t}\right)_{-T(\mu) \leq t<\infty}$ converges locally in $C^{1}$ to $\left(G_{t}\right)_{-\infty<t<\infty}$ which is the weak solution of (44) with the single point $\{x\}$ as the initial condition.

Let $W^{\epsilon}$ be defined by (요), and

$$
\left.V_{\epsilon}(t)=\operatorname{Vol}\left(\left\{(x, z) \in \Omega_{L} \times \mathbb{R}: W^{\epsilon}(x, z)<t, \quad|z| \leq \frac{1}{2}\right)\right\}\right) .
$$

Note that the level sets of $W^{\epsilon}$ form a classical solution to (4). We see that $V_{\epsilon}(t)$ is a smooth function of $t$, and further more, we have the following result 
Lemma 3.1. Let $\chi_{\left\{|z| \leq \frac{1}{2}\right\}}(x, z)$ be the characteristic function of the domain $\mathbb{D}=\left\{(x, z) \in \Omega_{L} \times \mathbb{R}:|z| \leq \frac{1}{2}\right\}$. Then

$$
\frac{d V_{\epsilon}}{d t}=\int_{N_{t}^{\epsilon}} H_{\epsilon}^{-1} \chi_{\left\{|z| \leq \frac{1}{2}\right\}}(x, z) d S>0 .
$$

Here and in the sequel, $H_{\epsilon}$ denotes the mean curvature of $N_{t}^{\epsilon}$ in $\mathbb{D}$ with respect to the unit normal direction $\frac{\nabla W^{\epsilon}}{\left|\nabla W^{\epsilon}\right|}$.

Proof. Using the Co-area formula, we see that

$$
\begin{aligned}
V_{\epsilon}(t) & =\int_{\mathbb{D}} \chi_{\left\{|z| \leq \frac{1}{2}\right\}}(x, z) \chi_{\left\{W^{\epsilon}<t\right\}}(x, z) d v \\
& =\int_{-\infty}^{\infty} \int_{\left\{W^{\epsilon}=\sigma\right\}} \frac{\chi_{\left\{|z| \leq \frac{1}{2}\right\}}(x, z) \chi_{\left\{W^{\epsilon}<t\right\}}(x, z)}{\left|\nabla W^{\epsilon}\right|} d S d \sigma \\
& =\int_{-\infty}^{t} \int_{\left\{W^{\epsilon}=\sigma\right\}} \frac{\chi_{\left\{|z| \leq \frac{1}{2}\right\}}(x, z)}{\left|\nabla W^{\epsilon}\right|} d S d \sigma
\end{aligned}
$$

which implies

$$
\frac{d V_{\epsilon}}{d t}=\int_{N_{t}^{\epsilon}} H_{\epsilon}^{-1} \chi_{\left\{|z| \leq \frac{1}{2}\right\}}(x, z) d S>0 .
$$

This finishes the proof of the lemma.

A direct conclusion of Lemma 3.1 is the following

Corollary 3.2. Let $W^{\epsilon}$ be a classical solution to (4) on $\mathbb{D}$ and $v=\operatorname{Vol}(\{(x, z) \in$ $\left.\left.\Omega_{L} \times \mathbb{R}: W^{\epsilon}(x, z)<t, \quad|z| \leq \frac{1}{2}\right\}\right)$. Then $t$ is a smooth function of $v$ and

$$
\begin{aligned}
\frac{d t}{d v} & =\left(\int_{N_{t}^{\epsilon}} H_{\epsilon}^{-1} \chi_{\left\{|z| \leq \frac{1}{2}\right\}}(x, z) d S\right)^{-1} \\
& =\left(\int_{N_{t}^{\epsilon} \cap\left\{|z| \leq \frac{1}{2}\right\}} H_{\epsilon}^{-1} d S\right)^{-1} .
\end{aligned}
$$

Let $\left(G_{t}\right)_{t>-\infty}$ be the weak solution of (4). We have the following

Lemma 3.3. For any $v>0$ either there is a time $t \in \mathbb{R}$ with $\operatorname{Vol}\left(G_{t}\right)=v$ or $v$ is a jump volume for (4), i.e. there exists a time $t_{1}>-\infty$ with

$$
\operatorname{Vol}\left(G_{t_{1}}\right)<v \leq \operatorname{Vol}\left(G_{t_{1}}^{+}\right),
$$

where $G_{t_{1}}^{+}$is the strictly minimizing hull for $G_{t_{1}}$. 
Proof. Let

$$
t_{0}=\inf \left\{t \in \mathbb{R}: \operatorname{Vol}\left(G_{t}\right) \geq v\right\}
$$

and

$$
\tau_{0}=\sup \left\{t \in \mathbb{R}: \operatorname{Vol}\left(G_{t}\right) \leq v\right\} .
$$

Note that $t_{0} \geq \tau_{0}$. By [8], we know that $K_{t}=\partial G_{t}$ converges to $K_{t_{0}}^{+}$locally in $C^{1}$ when $t$ decreases to $t_{0}$ and $K_{t}$ converges to $K_{\tau_{0}}$ locally in $C^{1}$ when $t$ increases to $\tau_{0}$ so that $\operatorname{Vol}\left(G_{t_{0}}^{+}\right) \geq v \geq \operatorname{Vol}\left(G_{\tau_{0}}\right)$. If $t_{0}>\tau_{0}$, this contradicts the definition of $t_{0}$ or $\tau_{0}$. Thus $t_{0}=\tau_{0}$. Thus either $v$ satisfies $\operatorname{Vol}\left(G_{t_{0}}\right)=v$ or $\operatorname{Vol}\left(G_{t_{0}}\right)<v \leq \operatorname{Vol}\left(G_{t_{0}}^{+}\right)$. This finishes the proof of Lemma 3.3.

The next lemma is on the relation between $t$ and the volumes of the level sets of a weak solution of (41).

Lemma 3.4. For any $v>0$, let

$$
t(v)=\inf \left\{\tau: \operatorname{Vol}\left(G_{\tau}\right) \geq v\right\} .
$$

Then $t$ is a Lipschitz function and

$$
\frac{d t}{d v} \leq\left(\int_{K_{t}} H^{2}\right)^{\frac{1}{2}} \cdot\left(\operatorname{Area}\left(K_{t}\right)\right)^{-\frac{3}{2}}
$$

where $K_{t}=\partial G_{t}$.

Proof. For any fixed $v>0$, let $t^{i}(v)=t^{i}$ with $v=\operatorname{Vol}\left(\left\{(x, z) \in \Omega_{L} \times \mathbb{R}\right.\right.$ : $\left.\left.W^{\epsilon}(x, z)<t^{i}, \quad|z| \leq \frac{1}{2}\right\}\right)$. Then by Lemma 2.1, we see that $t^{i}(v)$ converges to $t(v)$. (Here we assume without loss of generality the initial condition $B_{\mu}(x)$ shrinks to $\{x\}$ as $i \rightarrow \infty$.) Next, according to Corollary 3.2

$$
\begin{aligned}
\frac{d t^{i}}{d v} & =\left(\int_{N_{t}^{i} \cap\left\{|z| \leq \frac{1}{2}\right\}} H_{i}^{-1} d S\right)^{-1} \\
& \leq\left(\int_{N_{t}^{i} \cap\left\{|z| \leq \frac{1}{2}\right\}} H_{i}^{2} d S\right)^{\frac{1}{2}}\left(\operatorname{Area}\left(N_{t}^{i} \cap\left\{|z| \leq \frac{1}{2}\right\}\right)\right)^{-\frac{3}{2}}
\end{aligned}
$$

Hence, for any $v_{1} \geq v_{2}$, we have

$$
t^{i}\left(v_{1}\right)-t^{i}\left(v_{2}\right) \leq \int_{v_{2}}^{v_{1}}\left(\int_{N_{t}^{i} \cap\left\{|z| \leq \frac{1}{2}\right\}} H_{i}^{2} d S\right)^{\frac{1}{2}}\left(\operatorname{Area}\left(N_{t}^{i} \cap\left\{|z| \leq \frac{1}{2}\right\}\right)\right)^{-\frac{3}{2}} d v
$$

According to (5.6) in [8], we see that for any $T>-T(\mu)$ and all $t \in$ $[-T(\mu), T]$

$$
\int_{N_{t}^{i} \cap\left\{|z| \leq \frac{1}{2}\right\}} H_{i}^{2} d S \leq C(T)
$$


here $C(T)$ is a constant that depends only on $T$. Using also (5.12) in [8] we see that for almost every $t>-T(\mu)$, we have

$$
\int_{N_{t}^{i} \cap\left\{|z| \leq \frac{1}{2}\right\}} H_{i}^{2} d S \rightarrow \int_{\tilde{N}_{t} \cap\left\{|z| \leq \frac{1}{2}\right\}} H^{2} d S .
$$

Letting $i \rightarrow \infty$ and using the bounded convergence theorem, we see that

$$
\begin{aligned}
t\left(v_{1}\right)-t\left(v_{2}\right) & \leq \int_{v_{2}}^{v_{1}}\left(\int_{\tilde{N}_{t} \cap\left\{|z| \leq \frac{1}{2}\right\}} H^{2}\right)^{\frac{1}{2}}\left(\operatorname{Area}\left(\tilde{N}_{t} \cap\left\{|z| \leq \frac{1}{2}\right\}\right)\right)^{-\frac{3}{2}} d v \\
& =\int_{v_{2}}^{v_{1}}\left(\int_{K_{t}} H^{2}\right)^{\frac{1}{2}} \cdot\left(\operatorname{Area}\left(K_{t}\right)\right)^{-\frac{3}{2}} d v
\end{aligned}
$$

This finishes the proof of the lemma.

Let $M_{\text {ext }}$ be the exterior region of $\left(M^{3}, g\right)$ defined in Lemma 4.1 in [8]. Let $\Omega \subset M$ be a Borel set with finite perimeter, and $\Omega_{e x t}=\Omega \cap M_{\text {ext }}$, here and in the sequel $M_{\text {ext }}$ is the exterior region of $M$, for the its definition see Lemma 4.1, P.392, 8]. Let

$$
A_{\text {ext }}(v)=\inf \left\{\mathcal{H}^{2}\left(\partial^{*} \Omega_{\text {ext }}\right): \mathcal{L}^{3}\left(\Omega_{\text {ext }}\right)=v\right\}
$$

Clearly, we have $A(v) \leq A_{\text {ext }}(v)$. In order to prove Theorem 1.2 and Theorem 1.3, we need $A_{\text {ext }}(v)$ to be nondecreasing:

Lemma 3.5. Let $\left(M^{3}, g\right)$ be an AF manifold with nonnegative scalar curvature. Let $M_{\text {ext }}$ be the exterior region of $M$, then $A_{\text {ext }}(v)$ is nondecreasing.

We will use an idea from [1] to prove this lemma: we need to construct a compact manifold with compact boundary from $M_{e x t}$. More precisely, note that $\left(M^{3}, g\right)$ is $\mathrm{AF}$, hence we may take a large compact domain $\Omega \subset M_{\text {ext }}$ so that $M_{\text {ext }} \backslash \Omega$ is diffeomorphic to $\mathbb{R}^{3} \backslash \mathbb{B}_{R+4}$, hence, for simplicity, we just assume $\Omega \backslash K$ is differmorphic to $\mathbb{B}_{R+4} \backslash \mathbb{B}_{\frac{R}{2}}$, here $K$ is the compact domain of $M$ which appears in Definition 1.1. On the other hand, we observe that the standard sphere with radius $\frac{\lambda}{2}$ can be expressed as $\mathbb{S}^{2}(\lambda)=\left(\mathbb{R}^{3}, g_{S}=\right.$ $\left.\frac{\left(d x^{1}\right)^{2}+\left(d x^{2}\right)^{2}+\left(d x^{3}\right)^{2}}{\left(1+\lambda^{-2}|x|^{2}\right)^{2}}\right)$. Let

$$
\bar{g}=\left\{\begin{array}{l}
g, \quad \text { inside } \mathbb{B}_{R+5} \\
\eta g+(1-\eta) g_{S}, \text { on } \mathbb{B}_{R+6} \backslash \mathbb{B}_{R+5} \\
g_{S}, \quad \text { outside } \mathbb{B}_{R+6}
\end{array}\right.
$$

where $\eta$ is a smooth function with $\eta=1$ in $\mathbb{B}_{R+5}$ and that vanishes outside $\mathbb{B}_{R+6}$. Thus $\left(M_{\text {ext }}, \bar{g}\right)$ can be regarded as a compact manifold with compact boundary. We denote this manifold by $(\bar{M}, \bar{g})$.

We also need the following result from [10, Lemma 1]. 
Lemma 3.6. [Meeks-Yau] Let $\iota$ be the infinmum of the injectivity radius of points in $\left\{x \in \bar{M} \mid d\left(x, S_{\frac{R}{2}}\right)>\frac{d}{4}\right\}$. Let $K>0$ be the upper bound of the curvature of $\bar{M}$ outside $\mathbb{B}_{\frac{R}{2}}$. Let $S_{\frac{R}{2}}$ be the coordinate sphere with radius $\frac{R}{2}$, suppose $N$ is a minimal surface and suppose $x \in N$ is a point satisfying $d\left(x, S_{\frac{R}{2}}\right) \geq \frac{d}{2}$, then

$$
\left|N \cap B_{x}(r)\right| \geq 2 \pi K^{-2} \int_{0}^{r} \tau^{-1}(\sin K \tau)^{2} d \tau
$$

where $r=\min \left\{\frac{d}{4}, \iota\right\}$.

Proof of Lemma 3.5. Assume that $A_{\text {ext }}(v)$ is not nondecreasing. Then there are $v_{1}<v_{2}$ with $A_{e x t}\left(v_{1}\right)>A_{\text {ext }}\left(v_{2}\right)$. Using result from geometry measure theory ([12]), there is a compact domain $\Omega_{0} \subset \bar{M}$ with compact boundary $\Sigma_{0}$ and $\Sigma_{0} \backslash \partial M_{e x t}$ is smooth, and

$$
\operatorname{Area}_{\bar{g}}\left(\Sigma_{0}\right)=\inf \left\{\operatorname{Area}_{\bar{g}}(\partial \Omega): \Omega \subset \bar{M}, \operatorname{Vol}_{\bar{g}}(\Omega) \geq v_{1}\right\} .
$$

Here and in the sequel $A r e a_{\bar{g}}$, and $V o l_{\bar{g}}$ denote area and volume with respect to metric $\bar{g}$ respectively. We claim that $\operatorname{Vol}_{\bar{g}}\left(\Omega_{0}\right)>v_{1}$ provided $R$ and $\lambda$ are large enough. Therefore, $\Sigma_{0} \backslash \partial M_{\text {ext }}$ is a stable minimal surface in $\bar{M}$. In fact, suppose $\operatorname{Vol}_{\bar{g}}\left(\Omega_{0}\right)=v_{1}$, for any $\epsilon>0$, we assume there is a compact domain $\mathbb{D}_{2} \subset M$ with $\operatorname{Vol}_{g}\left(\mathbb{D}_{2}\right)=v_{2}$ and Areag $\left(\partial \mathbb{D}_{2}\right)<A_{\text {ext }}\left(v_{2}\right)+\epsilon$, and without loss of generality, we assume $\mathbb{D}_{2}$ is contained in $\Omega$, then we have

$$
\operatorname{Area}_{\bar{g}}\left(\Sigma_{0}\right) \leq \operatorname{Area}_{\bar{g}}\left(\partial \mathbb{D}_{2}\right)=\operatorname{Area}_{g}\left(\partial \mathbb{D}_{2}\right)<A_{\text {ext }}\left(v_{2}\right)+\epsilon<A_{\text {ext }}\left(v_{1}\right),
$$

which implies $\Omega_{0}$ cannot be contained in $\Omega$ completely.

If $\Omega_{0}$ is contained the domain outside $\mathbb{B}_{R+6}$, then by the solution of isoperimetric problem on the standard sphere, we see that when $R$ and $\lambda$ become large enough, the diameter of $\Omega_{0}$ in $\bar{M}$ is uniform bounded independently of $R$ and $\lambda$. However, for any fixed $R$, taking $\lambda$ large enough, we see that the metric $\bar{g}$ restricted on $\Omega_{0}$ is almost Euclidean. Then, by a translation in $\mathbb{R}^{3}$, we may find a domain $\Omega_{1}$ which is contained in $\mathbb{B}_{R} \backslash \mathbb{B}_{\frac{R}{2}} \subset \Omega$ and isometric to $\Omega_{0}$ in $\mathbb{R}^{3}$. Hence, the volume of $\Omega_{1}$ and area of the boundary of $\Omega_{1}$ are very close to those of $\Omega_{0}$ with respect to metric $\bar{g}$ provided $R$ and $\lambda$ is large enough. By a small perturbation on $\Omega_{1}$ if necessary, we may assume $\operatorname{Vol}_{\bar{g}}\left(\Omega_{1}\right)=\operatorname{Vol}_{\bar{g}}\left(\Omega_{0}\right)$, and $A_{\text {ext }}\left(v_{1}\right) \leq \operatorname{Area}_{\bar{g}}\left(\partial \Omega_{1}\right) \leq \operatorname{Area}_{\bar{g}}\left(\Sigma_{0}\right)+\epsilon$, which is contradiction to (14), provided that $\epsilon>0$ is sufficiently small.

For the remaining case, by the co-area formula, we see that we may find a coordinate sphere $S_{\rho}$ with Area $_{\bar{g}}\left(S_{\rho} \cap \Omega_{0}\right)<\epsilon$, and $R+6 \leq \rho \leq 2 R$. By the solution of the classical isoperimetric problem on the standard sphere, we may assume the diameter of the part of $\Omega_{0}$ which outside $\mathbb{B}_{\rho}$ has uniform bounded 
independently of $R$ and $\rho$. Therefore, by the same reasoning as above, we may translate the part of $\Omega_{0}$ which outside $\mathbb{B}_{\rho}$ into $\mathbb{B}_{R} \backslash \mathbb{B}_{\frac{R}{2}}$ completely and get a new domain denoted by $\Omega_{2}$ which may have several connected components and contained in $\mathbb{B}_{R}$. Note that $g$ is asymptotically flat. We see that the volume of $\Omega_{2}$ and area of the boundary of $\Omega_{2}$ with respect to $g$ are very close to these of $\Omega_{0}$. By a perturbation of $\Omega_{2}$ if necessary, we get a domain in $\Omega$ which is still denoted by $\Omega_{2}$ with $\operatorname{Vol}_{\bar{g}}\left(\Omega_{2}\right)=\operatorname{Vol}_{g}\left(\Omega_{2}\right)=v_{1}$. We again get $A_{\text {ext }}\left(v_{1}\right) \leq \operatorname{Area}_{\bar{g}}\left(\partial \Omega_{2}\right) \leq \operatorname{Area}_{\bar{g}}\left(\Sigma_{0}\right)+2 \epsilon$, which is contradiction to (14), provided $\epsilon>0$ is small enough. Therefore, $\operatorname{Vol}_{\bar{g}}\left(\Omega_{0}\right)>v_{1}$, and hence, as we claimed before, $\Sigma_{0} \backslash \partial M_{e x t}$ is a stable minimal surface in $\bar{M}$.

Finally, we want to prove the minimal surface $\Sigma_{0}$ is contained in $\mathbb{B}_{R+1}$ when $R$ is large enough. In particular, it is in $\Omega$. Actually, for any $x \in \Sigma_{0} \backslash \mathbb{B}_{R+1}$, note that $(M, g)$ is AF. Thus, we may assume that $\iota>\frac{R}{2}$ and $K \leq C R^{-3}$ outside $\mathbb{B}_{R+1}$. By (13), we have that

$$
\operatorname{Area}_{\bar{g}}\left(\Sigma_{0}\right) \geq C R^{2}
$$

where $C$ is a constant independent of $R$. However, by (14), we see that this is a contradiction when $R$ is sufficiently large. Thus, $\Sigma_{0} \backslash \partial M_{e x t}$ is contained in $\Omega$. Without loss of generality, we may assume that $M_{e x t}$ is foliated by spheres of positive mean curvature. It follows there are no minimal surfaces contained in $M_{\text {ext }}$. This finishes proof of the lemma.

Now, we can prove Theorem 1.2 and Theorem 1.3 .

Proof of Theorem 1.2 and Theorem 1.3. For any $v>0$, we may choose a sufficiently large $\rho=\rho(v)$, for any $x \in M \backslash B_{\rho}(0) \subset M_{\text {ext }}$ and consider the IMCF (44) with initial condition $\{x\}$. By choosing $\rho>0$ sufficiently large if necessary, we may assume there is a $G_{t}$ which is a domain in the weak solution of (44) with initial condition $\{x\}$, satisfying $\operatorname{Vol}\left(G_{t}\right)>v$, and $G_{t}$ is contained in the interior part of $M_{\text {ext }}$. By a direct computation and Lemma 3.4 , we see that

$$
\frac{d B}{d v} \leq B^{-\frac{1}{2}}\left(\int_{K_{t}} H^{2}\right)^{\frac{1}{2}} .
$$

By the definition of the Hawking mass of $K_{t(v)}$, we see that

$$
\int_{K_{t}} H^{2}=16 \pi-(16 \pi)^{\frac{3}{2}} B^{-\frac{1}{2}} m(v),
$$

In conjunction with previous inequality, we obtain (see also Proposition 3 in [2]). 


$$
B(v) \leq(36 \pi)^{\frac{1}{3}}\left(\int_{0}^{v}\left(1-(16 \pi)^{\frac{1}{2}} B^{-\frac{1}{2}}(t) m(t)\right)^{\frac{1}{2}} d t\right)^{\frac{2}{3}} .
$$

If $v$ is not a jump volume, then there is a $G_{t}$ with $\operatorname{Vol}\left(G_{t}\right)=v$ so that in this case we have

$$
\begin{aligned}
A(v) & \leq A_{\text {ext }}(v) \leq \operatorname{Area}\left(K_{t}\right)=B(v) \\
& \leq(36 \pi)^{\frac{1}{3}}\left(\int_{0}^{v}\left(1-(16 \pi)^{\frac{1}{2}} B^{-\frac{1}{2}}(t) m(t)\right)^{\frac{1}{2}} d t\right)^{\frac{2}{3}} ;
\end{aligned}
$$

If $v$ is a jump volume, then there is a $G_{\tau}$ with $v_{1}=\operatorname{Vol}\left(G_{\tau}\right)<v \leq$ $\operatorname{Vol}\left(G_{\tau}^{+}\right)=v_{2}$. Hence $t(v)=\tau$ and thus $B(v)=B\left(v_{1}\right)$,

$$
A(v) \leq A_{\text {ext }}(v) \leq A_{\text {ext }}\left(v_{2}\right) \leq \operatorname{Area}\left(K_{\tau}^{+}\right)=\operatorname{Area}\left(K_{\tau}\right)=B\left(v_{1}\right)=B(v) .
$$

Here we have used Lemma 3.5 in the first inequality. This finishes proof of Theorem 1.2 .

Suppose there is a $v_{0}>0$ with $A\left(v_{0}\right)=(36 \pi)^{\frac{1}{3}} v_{0}^{\frac{2}{3}}$. We claim that in this case $v_{0}$ is not a jump volume. Suppose not, then we may find $v_{1}, v_{2}$ with $v_{1}<v_{0} \leq v_{2}$, and $\operatorname{Vol}\left(G_{t_{1}}\right)=v_{1}$ and $\operatorname{Vol}\left(G_{t_{1}}^{+}\right)=v_{2}$. Since $A_{\text {ext }}(v)$ is nondecreasing, we see that $A_{\text {ext }}\left(v_{1}\right) \leq A_{\text {ext }}\left(v_{0}\right) \leq A_{\text {ext }}\left(v_{2}\right)$. However,

$$
\begin{gathered}
A_{\text {ext }}\left(v_{1}\right) \leq \operatorname{Area}\left(K_{t_{1}}\right) \leq(36 \pi)^{\frac{1}{3}} v_{1}^{\frac{2}{3}}, \\
A\left(v_{0}\right)=(36 \pi)^{\frac{1}{3}} v_{0}^{\frac{2}{3}}, \\
A_{\text {ext }}\left(v_{2}\right) \leq \operatorname{Area}\left(K_{t_{1}}^{+}\right)=\operatorname{Area}\left(K_{t_{1}}\right) .
\end{gathered}
$$

Combine these inequalities, we see that $v_{0} \leq v_{1}$, which is a contradiction. Thus $v_{0}$ is not a jump volume.

Suppose there is non-flat point $x \in M \backslash B_{\rho}(0)$. We consider the weak solution of (44) with initial condition $\{x\}$. By Lemma 8.1 in [8], $m(v)>0$, for $v>0$, together with (15), we that there is $t>-\infty$ with $\operatorname{Vol}\left(G_{t}\right)=v_{0}$. Thus,

$$
A\left(v_{0}\right) \leq B\left(v_{0}\right)<(36 \pi)^{\frac{1}{3}} v_{0}^{\frac{2}{3}},
$$

which is a contradiction. Thus $M \backslash B_{\rho}(0)$ is flat. It follows that the ADM mass of $(M, g)$ is zero so that $(M, g)$ is flay by the positive mass theorem proved in [11], we see $(M, g)=\mathbb{R}^{3}$. This finishes proof of Theorem 1.3. 
Yuguang Shi

\section{REFERENCES}

[1] H.L. Bray, The Penrose inequality in general relativity and volume comparison theorems involving scalar curvature (thesis), [math.DG] (1998).

[2] S. Brendle \& O. Chodosh, A volume comparison theorem for asymptotically hyperbolic manifolds, Comm. Math. Phys. 332 (2014), no. 2, 839-846. MR 3257665

[3] A.Carlotto, O. Chodosh \& M. Eichmair Efficitive versions of the positive mass theorem, preprint, http://arxiv.org/abs/1503.05910v1 (2015).

[4] D. Christodoulou \& S.-T. Yau, Some remarks on the quasi-local mass, Mathematics and general relativity (Santa Cruz, CA, 1986), Contemp. Math., vol. 71, Amer. Math. Soc., Providence, RI, 1988, pp. 9-14, MR 954405.

[5] M. Eichmair \& J. Metzger, Large isoperimetric surfaces in initial data sets, J.Differential Geom. Vol.94 (2013) 159-186, MR 3031863

[6] M. Eichmair \& J. Metzger, Unique isoperimetric foliations of asymptotically flat manifolds in all dimensions, Invent. Math. Vol.194 (2013) 591-630, MR 3127063

[7] X.-Q. Fan, Y.-S. Shi \& L.-F. Tam, Large-sphere and small-sphere limits of the BrownYork mass, Comm. Anal. Geom. 17 (2009), no. 1, 37-72, MR 2495833.

[8] G.Huisken \& T.Ilmanen The inverse mean curvature flow and the Riemannian Penrose Inequality. J.Differential Geom., 59 (2001) 353-437, MR 1916951

[9] G. Huisken, An isoperimetric concept for mass and quasilocal mass, Oberwolfach reports 3 (2006), no. 2, 87-88.

[10] W.H. Meeks \& S.-T. Yau, Topology of three-dimensional manifolds and the embedding problems in minimal surface theory, Ann. of Math. 112 (1980), 441-484, MR 595203

[11] R.Schoen \& S.-T. Yau, On the proof of the positive mass conjecture in General Relativity, Comm. Math. Phys 65 (1979), 45-76. MR 526976

[12] L. M. Simon, Lectures on Geometric Measure Theory, Proc. of Centre for Math. Analysis, 3. Austr. Nat. Univ., 1983

Yuguang Shi, Key Laboratory of Pure and Applied mathematics, School of Mathematical Sciences, Peking University, Beijing, 100871, P.R. China.

E-mail address: ygshi@math.pku.edu.cn 